\title{
How Detergent Impacts Membrane Proteins: Atomic-Level Views of Mitochondrial Carriers in Dodecylphosphocholine
}

Vilius Kurauskas, ${ }^{\dagger}$ Audrey Hessel, ${ }^{\dagger}$ Peixiang Ma, ${ }^{\dagger}$ Paola Lunetti, ${ }^{\dagger}$ Katharina Weinhäupl, ${ }^{\dagger}$ Lionel Imbert, $^{\dagger}$ Bernhard Brutscher, ${ }^{\dagger}$ (ఠ) Martin S. King, ${ }^{\S}$ Rémy Sounier, ${ }^{\prime}$ Vincenza Dolce, ${ }^{\perp}$ Edmund R. S. Kunji, ${ }^{\S}$ Loredana Capobianco, ${ }^{*}{ }^{\ddagger}$ Christophe Chipot, ${ }^{\# \odot ~ F r a n c ̧ o i s ~ D e h e z, ~}{ }^{*}, \#$ Beate Bersch, ${ }^{\dagger}$ and Paul Schanda ${ }^{*},{ }^{\dagger}$

${ }^{\dagger}$ Université Grenoble Alpes, CNRS, CEA, IBS, 38000 Grenoble, France

${ }^{\ddagger}$ Department of Biological and Environmental Sciences and Technologies, University of Salento, 73100 Lecce, Italy

${ }^{\S}$ MRC-MBU, University of Cambridge, Cambridge CB2 OXY, United Kingdom

"CNRS, INSERM, Université de Montpellier, 34094 Montpellier, France

${ }^{\perp}$ Dept of Pharmacy, University of Calabria, 87036 Arcavacata di Rende, Italy

\#LPCT, UMR 7019 Université de Lorraine, CNRS and Laboratoire International Associé \& University of Illinois at Urbana-Champaign, F-54500 Vandoeuvre-lès-Nancy, France

Supporting Information

ABSTRACT: Characterizing the structure of membrane proteins (MPs) generally requires extraction from their native environment, most commonly with detergents. Yet, the physicochemical properties of detergent micelles and lipid bilayers differ markedly and could alter the structural organization of MPs, albeit without general rules. Dodecylphosphocholine (DPC) is the most widely used detergent for MP structure determination by NMR, but the physiological relevance of several prominent structures has been questioned, though indirectly, by other biophysical techniques, e.g., functional/ thermostability assay (TSA) and molecular dynamics (MD) simulations. Here, we resolve unambiguously this controversy by probing the functional relevance of three different mitochondrial carriers (MCs) in DPC at the atomic level, using an exhaustive set of solution-NMR experiments, complemented by functional/TSA and MD data. Our results provide atomic-level insight into the structure, substrate interaction and dynamics of the detergent-membrane protein complexes and demonstrates cogently that, while high-resolution NMR signals can be obtained for MCs in DPC, they systematically correspond to nonfunctional states.

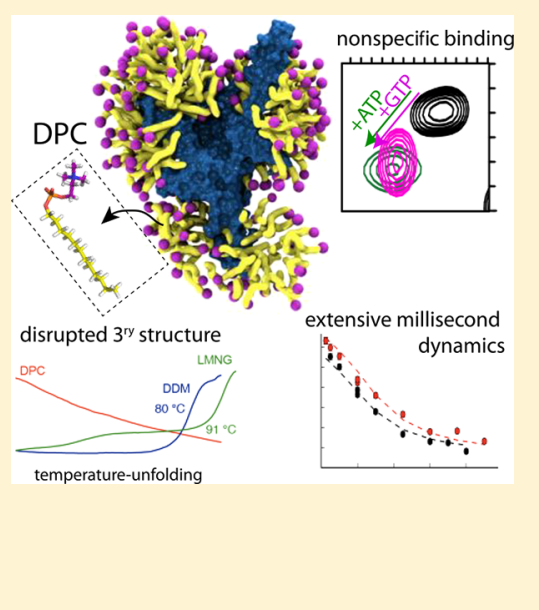

$\mathrm{M}$ tochondrial carriers (MCs) constitute a large family of transport proteins of ca. $30-35 \mathrm{kDa}$ molecular weight that play important roles in the intracellular translocation of metabolites, nucleotides, and coenzymes. ${ }^{1}$ The transport mechanism involves the alternating exposure of a central cavity to the cytosolic side (c-state) or the mitochondrial matrix (mstate). ${ }^{2}$ Crystallographic structures of the most prominent member, the adenosine diphosphate $(\mathrm{ADP}) /$ adenosine triphosphate (ATP) carrier (AAC), extracted from the native mitochondrial membrane ${ }^{3,4}$ could only be obtained in the presence of the strong inhibitor carboxyatractyloside (CATR), which locks the carrier in an aborted c-state. These structures revealed a central cavity, in which CATR is bound, surrounded by six transmembrane helices. No other atomic-resolution structures of MCs are available, underscoring the difficulty of obtaining crystals of these dynamic proteins. Solution-NMR may overcome the difficulties that crystallography faces for dynamic MPs. Substrate, lipid, and inhibitor binding ${ }^{5-10}$ as well as dynamics, ${ }^{8,11}$ detected by NMR have been reported and related to functional mechanisms of this class of membrane proteins. Other biophysical techniques, e.g., functional/thermo- stability assays and molecular-dynamics simulations, have challenged some of these interpretations. ${ }^{12-14}$ Solving this apparent controversy has been hampered by the lack of direct experimental atomic-resolution data and stringent control experiments. Here, we resort to an extensive set of NMR experiments to address the structure, substrate interactions and dynamics of MCs in dodecylphosphocholine (DPC) alongside with functional/thermostability assays and molecular-dynamics simulations and conclude on their physiological significance.

To probe the structural integrity of the yeast ADP/ATP carrier 3 (AAC3) in different detergents, we first investigated its thermal stability, consistently with our previous investigations. $^{13,14}$ We produced AAC3 in the yeast mitochondrial membrane, extracted it with the mild detergent dodecylmaltoside (DDM), diluted it into solutions of either DDM, lauryl maltoside neopentyl glycol (LMNG) or DPC, and performed

Received: January 26, 2018

Accepted: February 2, 2018

Published: February 3, 2018 
thermostability shift assay (TSA) experiments. ${ }^{14,15}$ In DDM and LMNG, a typical protein melting curve reveals a cooperative thermal denaturation event with an apparent melting temperature of ca. $49{ }^{\circ} \mathrm{C}$ (Figure 1, blue/green

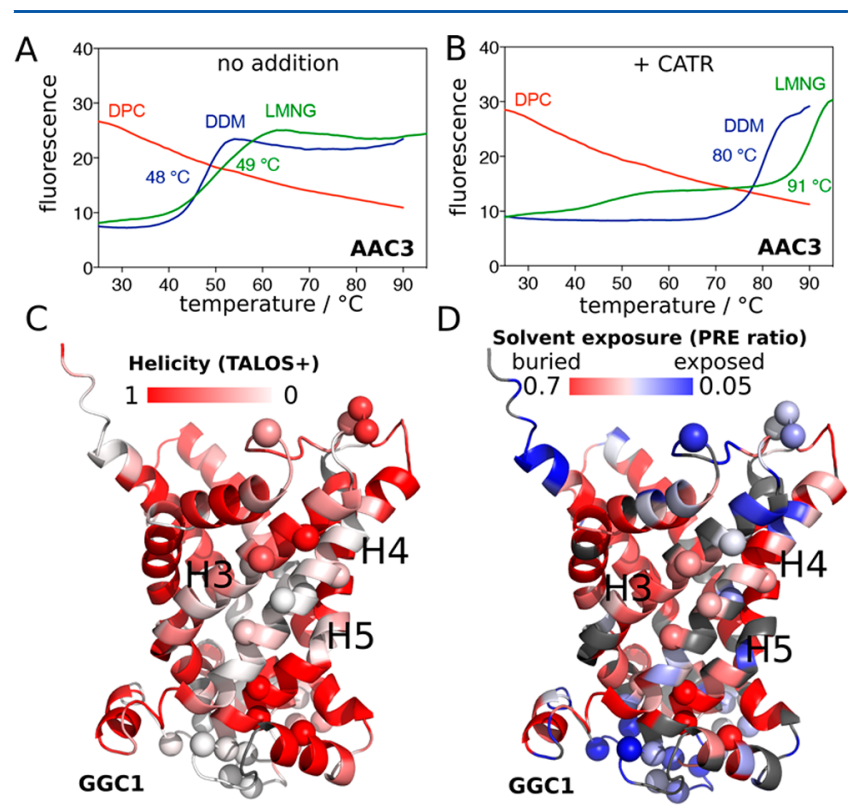

Figure 1. Stability of secondary and tertiary structures in MCs. (A, B) Thermostability of AAC3, extracted from yeast mitochondria and purified in DDM, diluted 20-fold into 0.1\% DDM (blue line), $0.1 \%$ LMNG (green line) or $0.1 \%$ DPC (red line) in the absence (A) or presence (B) of CATR, measured with TSA experiments (see Supplementary Methods). (C) Residue-wise helix propensity in GGC1, determined from NMR chemical shifts using the program TALOS $+{ }^{16}$ plotted onto a structural model of GGC1. Residues in white color are not in a helical conformation. (D) Residue-wise solvent accessibility in GGC1, as probed with the paramagnetic agent gadodiamide. Residues shown in white-to-blue colors are accessible to solvent. In panels $\mathrm{C}$ and $\mathrm{D}$, amide sites undergoing $\mu \mathrm{s}-\mathrm{ms}$ dynamics (discussed further below) are indicated by spheres.

lines). In the CATR-bound state, the melting temperature is dramatically increased to $80-90{ }^{\circ} \mathrm{C}$. In contrast, the same protein preparation diluted into DPC already shows a high fluorescence signal at low temperature, and no transition in the course of the temperature scan, irrespective of the presence of CATR. These observations indicate that AAC3 in DPC does not form a stable tertiary structure, nor binds CATR, in line with previous findings. ${ }^{13}$ To understand this behavior at the atomic level, we turned to solution-NMR of three members of the MC family, namely, AAC3, guanine diphosphate (GDP)/ guanosine triphosphate (GTP) carrier (GGC1), and ornithine carrier (ORC1). Given their low sequence identity $(<32 \%$, Figure S1), common traits observed in DPC are likely shared by the entire MC family. Samples of AAC3, GGC1, and ORC1, prepared through refolding from inclusion bodies, just like in previous NMR studies of MCs, ${ }^{5,7,8,11}$ are all homogeneous (as shown by size-exclusion chromatograms and analytical ultracentrifugation; Figure S2). Consistently with previous NMR studies, ${ }^{5-9,11}$ all these samples yield high-quality NMR spectra (Figure S3), a criterion that is commonly employed to assess the functional relevance of MP samples and motivate structural studies. To reconcile these data with TSA experiments and to characterize the structural organization of MCs that is responsible for high-quality NMR spectra, we performed an
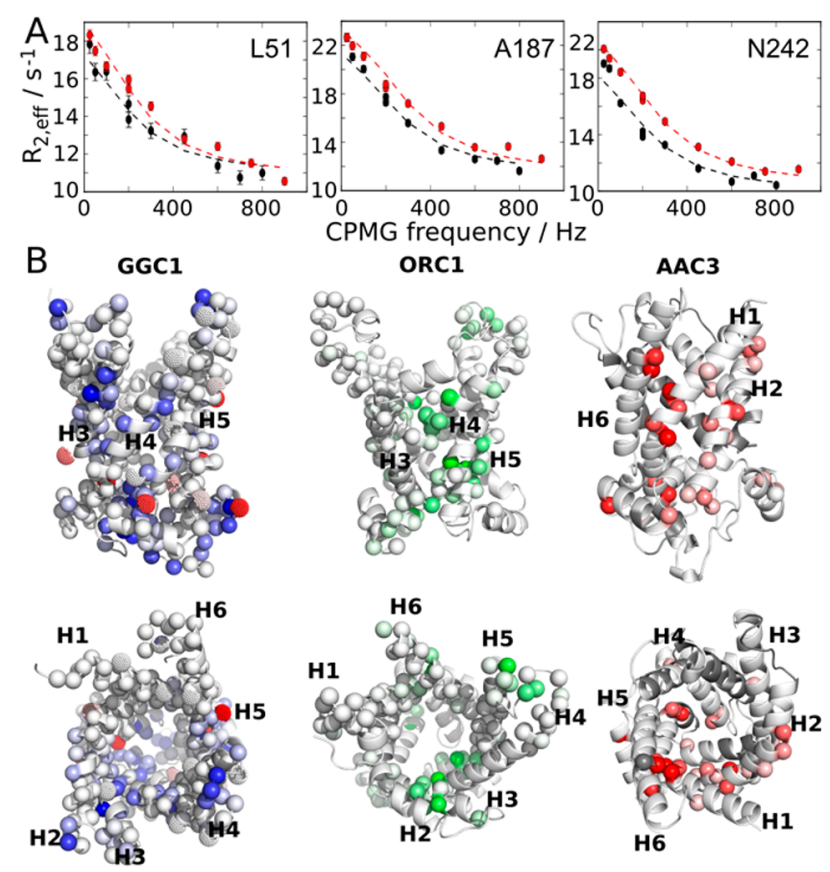

$\begin{array}{ll}\Delta R_{2, \text { eff }} & \mathrm{NH} 15=0 \mathrm{~s}^{-1} \\ \Delta \mathrm{R}_{2, \text { eff }} \mathrm{CH}_{3} 20 & 0 \mathrm{~s}^{-1}\end{array}$

C transport (liposomes) rel. transport activity

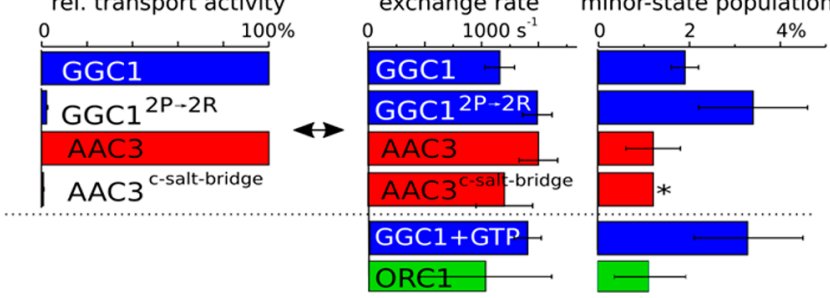
exchange rate minor-state population

Figure 2. Millisecond dynamics in MCs in DPC detergent micelles. (A) Representative 3D-HNCO-detected CPMG RD profiles of ${ }^{15} \mathrm{~N}$ amide sites in GGC1 (WT, no substrate). All nonflat RD profiles of this study are shown in Figures S7 to S17. (B) Location of residues with nonflat CPMG RD curves in GGC1, ORC1, and AAC3 plotted onto structural models based on the crystal structure of AAC3. For GGC1 and ORC1, the color code represents the extent of relaxationdispersion, as determined from the difference in $R_{2, \text { eff }}$ of the first and last points of the RD profiles; for AAC3, the color code represents $\Delta \omega$ values reported in ref 8 . A qualitatively similar picture is obtained for two mutants studied here, $\mathrm{GGC}^{2 \mathrm{P} \rightarrow 2 \mathrm{R}}$ and $\mathrm{AAC}^{-\mathrm{c} \text {-saltbridge }}$; see main text. (C) Transport activity and (D) NMR-derived dynamics parameters in MCs. The $p_{\mathrm{B}}$ value denoted with an asterisk was fixed to the value found for AAC3, which resulted in satisfactory fits. All transport activity and dynamics data reported in this study are provided in Tables S1-S3.

NMR-observed temperature series up to ca. $70{ }^{\circ} \mathrm{C}$ (Figure S3). One expects a discontinuity in the NMR parameters if collective unfolding arises. By contrast, we observe linear chemical-shift changes up to high temperatures, mirroring the TSA data, and suggesting that these MCs undergo a gradual change of the structural ensemble without defined denaturation transition.

We probed the structural and dynamical properties of MCs in DPC further with three different NMR observables. First, the residue-specific chemical-shift assignments were used with the TALOS+ software ${ }^{16}$ to derive local backbone geometry at the level of each residue (Figures 1C and S4). While there is an overall good agreement between the residues that, according to 


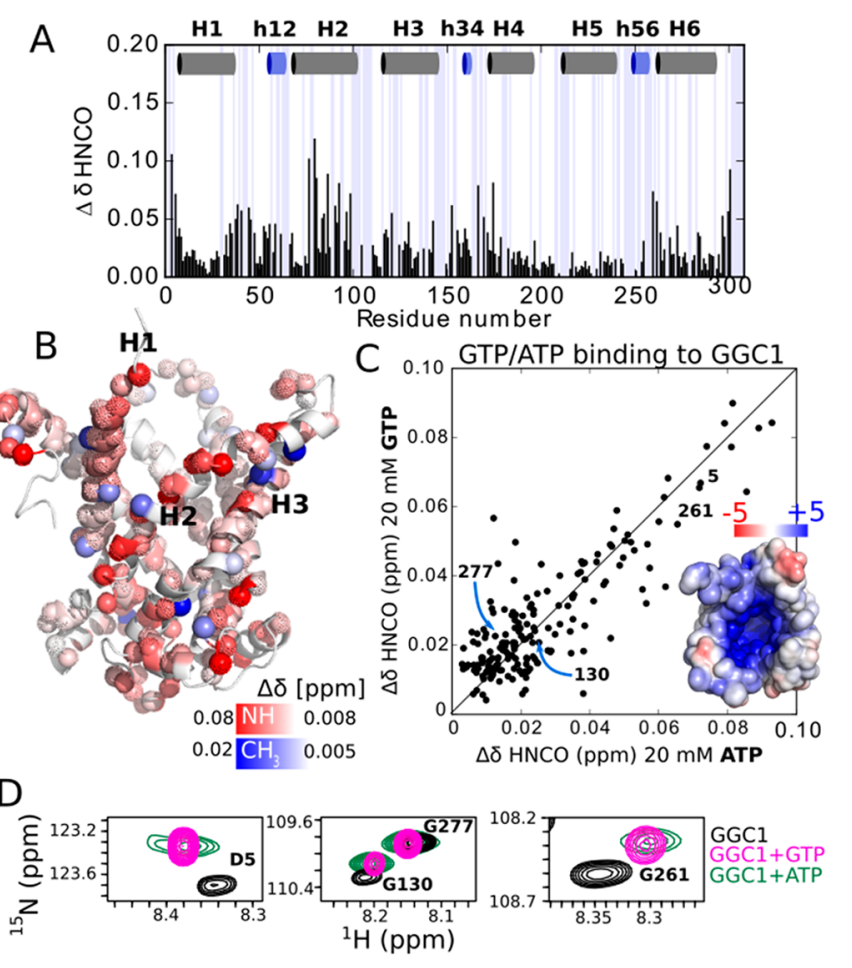

Figure 3. GGC1 in DPC lacks the expected binding specificity. (A) Chemical-shift perturbations in GGC1 upon addition of GTP. (B) Plot of amide $(\mathrm{H} / \mathrm{N} / \mathrm{CO})$ and methyl $(\mathrm{H} / \mathrm{C})$ CSPs onto a structural model of GGC1. (C,D) GTP and ATP produces very similar CSPs in GGC1, as exemplified with extracts from HNCO spectra (D) and shown as a correlation plot in (C). The inset shows the electrostatic surface of a AAC-derived structural model of GGC1. Equivalent data for AAC3 are shown in Figure S21.

their chemical shifts, have $\alpha$-helical conformation and the location of helices in the crystal structures of AAC3, several stretches of residues in the TM helices appear disordered in DPC, particularly in helices H4 (GGC1), and H2/H3 (AAC3). Independent support for the flexible nature of these parts comes from ${ }^{15} \mathrm{~N}$ transverse relaxation rate constants $\left(\mathrm{R}_{2}\right)$, which reveal enhanced flexibility in GGC1's H4 (Figure S5). Second, we performed solvent-paramagnetic relaxation-enhancement (sPRE) experiments with the soluble hydrophilic paramagnetic complex Gd-DTPA-BMA (gadodiamide), which probe the solvent-accessibility of each amide site (Figure 1D). As expected, the most exposed parts are located in loops of GGC1 and AAC3. Unexpectedly, however, sizable sPRE effects are also found in TM helices, in particular those for which TALOS+ finds a loop conformation. Third, we measured amide hydrogen/deuterium exchange (HDX) by dilution of GGC1 from $\mathrm{H}_{2} \mathrm{O}$ - into $\mathrm{D}_{2} \mathrm{O}$-buffer. The observed very fast $\mathrm{HDX}$ kinetics $(<1 \mathrm{~min})$ indicates that backbone hydrogen bonds are marginally stable (Figure S6).

To gain further insight into the properties and possibly functional relevance of MCs in DPC, we investigated the details of their dynamics. Functional turnover of MCs in the membrane occurs at a rate of ca. $500 \mathrm{~s}^{-1} .{ }^{17}$ Functional MCs are, thus, expected to undergo motions on this time scale, and these motions are expected to be sensitive to substrates or mutations. Carr-Purcell-Meiboom-Gill (CPMG) relaxationdispersion (RD) NMR experiments ${ }^{18}$ are ideally suited to probe $\mu \mathrm{s}-\mathrm{ms}$ motions at the level of individual residues. In this study we performed extensive CPMG RD measurements on a

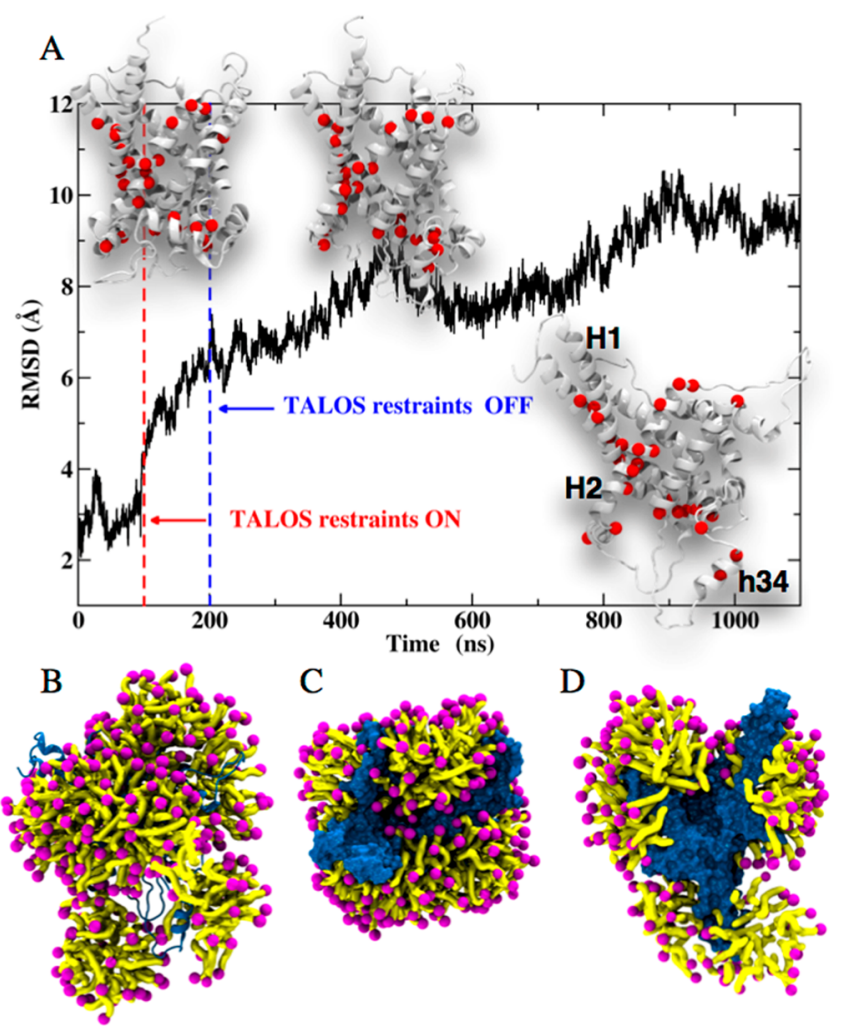

Figure 4. All-atom MD simulations of AAC3 in DPC micelles. (A) Time evolution of the root-mean-square displacement (RMSD) of yAAC3 backbone atomic positions with respect to those in the crystal structure. From 0 to $100 \mathrm{~ns}$, the protein embedded in DPC is free to move. From 100 to $200 \mathrm{~ns}$, a subset of dihedral angles is restrained to TALOS+ values inferred from NMR chemical shifts (see Materials and Methods and Table S4). At 200 ns, restraints are turned off. The secondary structures of the protein before and after applying the TALOS+ restraints and at the end of the trajectory are depicted chronologically in the insets. Amide sites for which $\mu \mathrm{s}-\mathrm{ms}$ motion is found in CPMG experiments are highlighted with red spheres. (B-D) Snapshots at $1 \mu \mathrm{s}$ simulation time showing the DPC (purple/yellow) organization around yAAC3 from side view (B), top view (C), and cut-open side view (D). Supplementary Movie 1 and Movie 2 show the time evolution of AAC3 and its surrounding micelle.

total of 10 different samples, including wild-type (WT) and mutant proteins, as well as samples with substrates and lipids, and on up to three magnetic field strengths per sample (see Table S1 and Figures S7-S17); in addition, previous work reported CPMG data for WT AAC $3 .{ }^{8}$ In AAC3, GGC1 and ORC1, we find extensive $\mu \mathrm{s}-\mathrm{ms}$ motions for about $20 \%$ of the ${ }^{15} \mathrm{~N}$ backbone amide sites, and in all cases the exchanging residues are clustered on one side of the molecule (Figure 2). We have performed additional ${ }^{13} \mathrm{C}$ CPMG experiments on methyl groups of Ala, Val, and Leu residues in GGC1 (Table S1 and Figure S8), independently confirming the presence of $\mu \mathrm{s}-$ ms dynamics in the region revealed by ${ }^{15} \mathrm{~N}$ CPMG experiments (red in Figure 2B, left). In order to obtain more insight into this process, we fitted a two-state exchange model to the CPMG RD data. Statistical analyses, shown in Figure S18, indicate that this simple model describes the data satisfyingly. The fitted parameters are similar for all three proteins, with an exchange rate constant, $k_{\mathrm{ex}}=k_{\mathrm{AB}}+k_{\mathrm{BA}}$ on the order of 1000-2000 s $\mathrm{s}^{-1}$, and populations of the minor state, $p_{\mathrm{B}}$, of ca. $1-3 \%$ (Figure $2 \mathrm{D}$ and Table S1). 
A major challenge in the interpretation of relaxation dispersion data is to understand the underlying motional process structurally. An intriguing observation is that the time scale of motion observed in all three MCs (ca. $1 \mathrm{~ms}$ ) is similar to the kinetics of transmembrane transport, ${ }^{17}$ suggesting a possible role in functional turnover. To test this hypothesis, we investigated whether the introduction of function-abolishing mutations or the presence of substrates and lipids alter the dynamics. We designed two mutants that address different structural/functional aspects of the mitochondrial carriers, reasoning that if they inactivate the proteins, they would do so for different reasons. In a first mutant, termed $\mathrm{GGC}^{2 \mathrm{P} \rightarrow 2 \mathrm{R}}$, two highly conserved Pro residues of the MC signature motifs (PX $[\mathrm{ED}] \mathrm{XX}[\mathrm{KR}]$ ) in $\mathrm{H} 3$ and $\mathrm{H} 5$ were mutated to Arg. Given the importance of these Pro kinks in the structure and mechanism, ${ }^{2}$ and the unfavorable energetics of placing Arg residues in the core of TM helices, ${ }^{19}$ we expected significant changes in the activity and dynamics. A second mutant targets Asp, Lys, and Gln residues on the cytosolic side of the TM helices of AAC3, which have been proposed to form a salt bridge network in the elusive $\mathrm{m}$-state. ${ }^{4}$ In the $\mathrm{AAC}^{-\mathrm{c} \text {-saltbridge }}$ mutant, we inverted charges such that the putative salt-bridge network cannot form any more. Liposome transport assays show that the residual transport activity in $\mathrm{GGC}^{2 \mathrm{P} \rightarrow 2 \mathrm{R}}$ and $\mathrm{AAC}^{-\mathrm{c} \text {-saltbridge }}$ is below $3 \%$, i.e., that the mutations render the proteins essentially nonfunctional (Figure 2C and Table S3).

Strikingly, however, the characteristics of the mutants in DPC micelles are hardly different from those of the WT protein samples. NMR spectra of these mutants are highly similar to the respective WT proteins. Chemical-shift differences are small, and restricted to residues in the immediate vicinity of the mutation sites (Figure S19). If the helices were in close contact to each other, as in the crystal structure, one would expect pronounced chemical-shift changes in the neighboring helices in these mutants. Another surprising observation is that the $\mu \mathrm{s}-\mathrm{ms}$ dynamics in these nonfunctional mutants is very similar to the one of WT proteins, even though the mutants are nonfunctional, likely suggesting that the $\mu \mathrm{s}-\mathrm{ms}$ motions are not directly related to function (Figures 2D, S12-S14 and Table S1). Along the same lines, we find that the presence of substrate (GTP) does not lead to a detectable difference in the dynamics compared to apo-GGC1 (Figure 2D), indicating that substrate cannot influence this dynamic process. Put together, the observed dynamics does not appear to be related to function. We reasoned that the functionally important ${ }^{2}$ lipid cardiolipin (CL) might resurrect the native properties of the protein. However, CPMG experiments with CL reveal an identical behavior for WT GGC1 and $\mathrm{GGCl}^{2 \mathrm{P} \rightarrow 2 \mathrm{R}}$ (see Supplementary Discussion and Figures S9, S11, and S20), showing that these MCs in DPC are nonfunctional, irrespective of added lipids.

An important criterion to assess functionality of a MP is its ability to interact with substrates. MCs are highly substratespecific transporters. In liposome transport assays, we find that recombinantly expressed and reconstituted AAC3 and GGC1 bind and transport specifically their substrate (ATP/GTP, respectively), while they do not bind the other nucleotide (GTP/ATP; see Table S3). Furthermore, CATR inhibits AAC3, but not GGC1 (see Supplementary Discussion). Testing the binding specificity is, thus, a convenient way to investigate the structural integrity of MCs in DPC. Addition of the substrate GTP to GGC1 leads to significant chemical shift perturbations (CSPs) for many amides and Ala, Leu, and Val methyls (Figure 3A). The largest CSPs are observed for residues lining $\mathrm{H} 2$, and on the cytoplasmic side of $\mathrm{H} 1$ and $\mathrm{H} 6$. Weaker yet significant effects are observed in the three amphipathic matrix helices (Figure 3B); these CSPs are in good agreement with previously reported data. ${ }^{11}$ However, addition of ATP, which does not interact with GGC1 in membranes, unexpectedly produces essentially the same CSPs as GTP (Figure 3C,D). Likewise, addition of GTP or ATP to AAC3 produces CSPs that are nearly identical to each other (Figure S21). Thus, while AAC3 and GGC1 in their lipidbilayer environment can discriminate between GTP and ATP, this ability is lost in DPC micelles. We repeated GTP-titration experiments with the nonfunctional GGC1 $1^{2 \mathrm{P} \rightarrow 2 \mathrm{R}}$ mutant. Even though this protein is nonfunctional and likely distorted through the Arg in the TM part, the GTP-induced CSPs are very similar to those observed in WT protein (Figure S22). This observation suggests that the observed GTP interaction is not dependent on structural integrity, and is thus nonspecific.

We then titrated WT GGC1 with CATR, a doubly negatively charged inhibitor of AAC, but not of GGC1. ${ }^{20}$ Despite the absence of GGC1/CATR interaction in membranes, the addition of CATR to GGC1 in DPC produces significant CSPs. The affected set of residues partly coincides with the sites that are modulated by the presence of GTP and ATP, and the effects are similar to those observed in AAC3 upon addition of CATR (Figure S23). These observations lead us to propose that the reported interactions of CATR with GGC1 or AAC3 in DPC are nonspecific and not reminiscent of binding in biological membranes. Indeed, the affinity of CATR to AAC3 in DPC $\left(K_{d}=20-150 \mu \mathrm{M}^{8}\right)$ is ca. 3 orders of magnitude weaker than the AAC/CATR interaction in lipid bilayers $\left(K_{d}=10-20\right.$ $\mathrm{nM}^{21}$ ) or of AAC extracted from mitochondria with LAPAO detergent $\left(K_{\mathrm{d}}=310 \mathrm{nM}^{21}\right)$. A plausible driving force for all these nonspecific low-affinity interactions is the electrostatic attraction between the negatively charged solutes (nucleotides, CATR) and the positively charged cavity of GGC1 and AAC3 (Figures 3C and S21D). In agreement with this view, ORC1, which has a significantly lower electrostatic charge, does not show significant CSPs upon addition of its substrate, Lornithine (Figure S24).

To obtain a mechanistic understanding of the structural organization of the protein and detergent, we turned to allatom molecular dynamics (MD) simulations in explicit DPC. $\mathrm{MD}$ simulations have been applied previously to MCs in a membrane environment ${ }^{22-24}$ and in DPC. ${ }^{12}$ We specifically sought a description of the structural organization of AAC3 in DPC, which would reflect our experimental data. In initial unrestrained all-atom simulations over $0.7 \mu \mathrm{s}$, AAC3 remains close to the conformation adopted in crystals, ${ }^{4}$ which is in disagreement with the TALOS-derived secondary structure in DPC. Presumably, the time scale accessible to simulation is too short to allow the transition of the compact conformation in crystals to the one in DPC micelles (see Supplementary Discussion and Figures S25, S26). To better reflect the experimental data, we, thus, used chemical-shift derived backbone torsional angles (limited to the $\phi, \psi$ angles for which the TALOS+ result was unambiguous; see Materials and Methods), and introduced a soft harmonic potential to these residues over a simulation period of $0.1 \mu \mathrm{s}$. This strategy, which rests on the use of NMR restraints to guide conformational analyses of proteins, is similar in spirit to that proposed by others. $^{25,26}$ When the torsional angles coincide with those inferred from the NMR data, the overall structure of AAC3 is 
markedly distorted, corresponding to a shift from ca. 3 to $6 \AA$ of the root-mean-square deviation (RMSD) of atomic positions (Figure 4A). The evolution of the tertiary structure upon introduction of the TALOS+ restraints is asymmetrical and chiefly involves the interfacial helix h34 (connecting $\mathrm{H} 3$ and $\mathrm{H} 4$ ) and the cytoplasmic side of $\mathrm{H} 1, \mathrm{H} 2$, and $\mathrm{H} 3$ (Figure 4 and S27). In a last step, the restraints were removed, and the simulation was extended to $1.1 \mu \mathrm{s}$. Over this time scale, AAC3 remains distorted. The cytoplasmic side of the TM helices, H1 and $\mathrm{H} 2$, and the interfacial helix h34 undergo relatively slow dynamics on the simulation time scale (see Movie 1). Interestingly, this region coincides with the segments that were found experimentally to undergo $\mu \mathrm{s}-\mathrm{ms}$ motions (red spheres in Figure 4). We speculate that the motions detected experimentally might correspond to the kind of slow rearrangements seen by MD. Most importantly, the simulations provide a view of how the detergent may organize around the protein. Figures $4 \mathrm{~B}-\mathrm{D}$ show that DPC molecules do not only form a corona around the hydrophobic core of the protein, as one might expect, but also form micelles interacting with both sides of the protein (cf. Movie 2). DPC molecules protrude between the TM helices and penetrate deep within the protein cavity (Figure 4C,D and Movie 2).

DPC has been employed for solving about $40 \%$ of all known NMR structures, but less than $1 \%$ of all crystal structures (Figure S28). Our data provide possible clues about the origin of this intriguing track record. In our hands, DPC is the only detergent able to maintain MCs in solution for extended time periods (a prerequisite for solution-NMR), and the embedded proteins exhibit well-resolved spectra and solute interactions that may be interpreted as signs of a functionally relevant sample. Our study reveals that these observations do not necessarily point to a functionally relevant protein sample, and stresses the importance of stringent control experiments.

\section{ASSOCIATED CONTENT}

\section{S Supporting Information}

The Supporting Information is available free of charge on the ACS Publications website at DOI: 10.1021/acs.jpclett.8b00269.

Movie 1: $1.1 \mathrm{~s}$ molecular-dynamics simulation of yeast AAC3 in DPC (MPG)

Movie 2: Same as Movie 1 including the explicit representation of DPC organization around yAAC3 (AVI)

Detailed experimental materials and methods, tables summarizing the results of transport activity measurements, CPMG fits and assigned chemical shifts, and figures with supporting experimental data (PDF)

\section{AUTHOR INFORMATION}

\section{Corresponding Authors}

*E-mail: paul.schanda@ibs.fr.

*E-mail: francois.dehez@univ-lorraine.fr.

*E-mail: loredana.capobianco@unisalento.it.

\section{ORCID}

Bernhard Brutscher: 0000-0001-7652-7384

Christophe Chipot: 0000-0002-9122-1698

Paul Schanda: 0000-0002-9350-7606

Notes

The authors declare no competing financial interest.

\section{ACKNOWLEDGMENTS}

We thank Klaus Zangger for providing gadodiamide, and Astrid C. Sivertsen, Guillaume Bouvignies, Eva Pebay-Peyroula, Stéphanie Ravaud, Ewen Lescop, Cécile Breyton, Sven Brüschweiler, Christine Ebel and Aline LeRoy for insightful discussions, Jason R. Schnell for help in preparing Figure S28 and Michael Kohlhaas for inspiring thoughts. This work was supported by the European Research Council (ERC-Stg311318) to P. S. and the UK Medical Research Council (U105663139) to E.R.S.K. This work used the platforms of the Grenoble Instruct Center (ISBG; UMS 3518 CNRS-CEAUJF-EMBL) with support from FRISBI (ANR-10-INSB-0502) and GRAL (ANR-10-LABX-49-01) within the Grenoble Partnership for Structural Biology (PSB).

\section{REFERENCES}

(1) Palmieri, F.; Monné, M. Discoveries, Metabolic Roles and Diseases of Mitochondrial Carriers: A Review. Biochim. Biophys. Acta, Mol. Cell Res. 2016, 1863, 2362-2378.

(2) Kunji, E. R. S.; Aleksandrova, A.; King, M. S.; Majd, H.; Ashton, V. L.; Cerson, E.; Springett, R.; Kibalchenko, M.; Tavoulari, S.; Crichton, P. G.; et al. The Transport Mechanism of the Mitochondrial ADP/ATP Carrier. Biochim. Biophys. Acta, Mol. Cell Res. 2016, 1863, 2379-2393.

(3) Pebay-Peyroula, E.; Dahout-Gonzalez, C.; Kahn, R.; Trezeguet, V.; Lauquin, G.; Brandolin, G. Structure of Mitochondrial ADP/ATP Carrier in Complex with Carboxyatractyloside. Nature 2003, 426, 3944.

(4) Ruprecht, J. J.; Hellawell, A. M.; Harding, M.; Crichton, P. G.; McCoy, A. J.; Kunji, E. R. S. Structures of Yeast Mitochondrial ADP/ ATP Carriers Support a Domain-Based Alternating-Access Transport Mechanism. Proc. Natl. Acad. Sci. U. S. A. 2014, 111, E426-34.

(5) Run, C.; Yang, Q.; Liu, Z.; OuYang, B.; Chou, J. J. Molecular Basis of MgATP Selectivity of the Mitochondrial SCaMC Carrier. Structure 2015, 23, 1394-1403.

(6) Zhao, L.; Wang, S.; Zhu, Q.; Wu, B.; Liu, Z.; OuYang, B.; Chou, J. J. Specific Interaction of the Human Mitochondrial Uncoupling Protein 1 with Free Long-Chain Fatty Acid. Structure 2017, 25, 13711379.e3.

(7) Zhao, L.; Wang, S.; Run, C.; OuYang, B.; Chou, J. J. Specific Lipid Binding of Membrane Proteins in Detergent Micelles Characterized by NMR and Molecular Dynamics. Biochemistry 2016, $55,5317-5320$.

(8) Brüschweiler, S.; Yang, Q.; Run, C.; Chou, J. J. SubstrateModulated ADP/ATP-Transporter Dynamics Revealed by NMR Relaxation Dispersion. Nat. Struct. Mol. Biol. 2015, 22, 636-641.

(9) Berardi, M. J.; Shih, W. M.; Harrison, S. C.; Chou, J. J. Mitochondrial Uncoupling Protein 2 Structure Determined by NMR Molecular Fragment Searching. Nature 2011, 476, 109-113.

(10) Berardi, M. J.; Chou, J. J. Fatty Acid Flippase Activity of UCP2 Is Essential for Its Proton Transport in Mitochondria. Cell Metab. 2014, 20, 541-552.

(11) Sounier, R.; Bellot, G.; Chou, J. J. Mapping Conformational Heterogeneity of Mitochondrial Nucleotide Transporter in Uninhibited States. Angew. Chem., Int. Ed. 2015, 54, 2436-2441.

(12) Zoonens, M.; Comer, J.; Masscheleyn, S.; Pebay-Peyroula, E.; Chipot, C.; Miroux, B.; Dehez, F. Dangerous Liaisons between Detergents and Membrane Proteins. The Case of Mitochondrial Uncoupling Protein 2. J. Am. Chem. Soc. 2013, 135, 15174-15182.

(13) Dehez, F.; Schanda, P.; King, M. S.; Kunji, E. R. S.; Chipot, C. Mitochondrial ADP/ATP Carrier in Dodecylphosphocholine Binds Cardiolipins with Non-Native Affinity. Biophys. J. 2017, 113, 23112315.

(14) Crichton, P. G.; Lee, Y.; Ruprecht, J. J.; Cerson, E.; Thangaratnarajah, C.; King, M. S.; Kunji, E. R. S. Trends in Thermostability Provide Information on the Nature of Substrate, 
Inhibitor, and Lipid Interactions with Mitochondrial Carriers. J. Biol. Chem. 2015, 290, 8206-8217.

(15) Alexandrov, A. I.; Mileni, M.; Chien, E. Y. T.; Hanson, M. A.; Stevens, R. C. Microscale Fluorescent Thermal Stability Assay for Membrane Proteins. Structure 2008, 16, 351-359.

(16) Shen, Y.; Delaglio, F.; Cornilescu, G.; Bax, A. TALOS+: A Hybrid Method for Predicting Protein Backbone Torsion Angles from NMR Chemical Shifts. J. Biomol. NMR 2009, 44, 213-223.

(17) Gropp, T.; Brustovetsky, N.; Klingenberg, M.; Muller, V.; Fendler, K.; Bamberg, E. Kinetics of Electrogenic Transport by the ADP/ATP Carrier. Biophys. J. 1999, 77, 714-726.

(18) Mittermaier, A. K.; Kay, L. E. Observing Biological Dynamics at Atomic Resolution Using NMR. Trends Biochem. Sci. 2009, 34, 601611.

(19) Zhou, H.-X.; Cross, T. A. Influences of Membrane Mimetic Environments on Membrane Protein Structures. Annu. Rev. Biophys. 2013, 42, 361-392.

(20) Vozza, A.; Blanco, E.; Palmieri, L.; Palmieri, F. Identification of the Mitochondrial GTP/GDP Transporter in Saccharomyces cerevisiae. J. Biol. Chem. 2004, 279, 20850-20857.

(21) Krämer, R.; Klingenberg, M. Reconstitution of Inhibitor Binding Properties of the Isolated Adenosine 5'-diphosphate, Adenosine 5'triphosphate Carrier-Linked Binding Protein. Biochemistry 1977, 16, 4954-49561.

(22) Wang, Y.; Tajkhorshid, E. Electrostatic Funneling of Substrate in Mitochondrial Inner Membrane Carriers. Proc. Natl. Acad. Sci. U. S. A. 2008, 105, 9598-9603.

(23) Dehez, F.; Pebay-Peyroula, E.; Chipot, C. Binding of ADP in the Mitochondrial ADP/ATP Carrier Is Driven by an Electrostatic Funnel. J. Am. Chem. Soc. 2008, 130, 12725-12733.

(24) Hedger, G.; Rouse, S. L.; Domanski, J.; Chavent, M.; Koldso, H.; Sansom, M. S. P. Lipid-Loving ANTs: Molecular Simulations of Cardiolipin Interactions and the Organization of the Adenine Nucleotide Translocase in Model Mitochondrial Membranes. Biochemistry 2016, 55, 6238-6249.

(25) Vallurupalli, P.; Hansen, D. F.; Kay, L. E. Structures of Invisible, Excited Protein States by Relaxation Dispersion NMR Spectroscopy. Proc. Natl. Acad. Sci. U. S. A. 2008, 105, 11766-11771.

(26) Robustelli, P.; Kohlhoff, K.; Cavalli, A.; Vendruscolo, M. Using NMR Chemical Shifts as Structural Restraints in Molecular Dynamics Simulations of Proteins. Structure 2010, 18, 923-933. 